\title{
Neurology and Neurosurgery in Nepal
}

\author{
Robert G. Lee and Dinesh N. Gongal
}

During early 1982 one of the authors (RGL) was privileged to spend two months in Nepal as part of an exchange program between the University of Calgary, Faculty of Medicine and the new medical school at Tribhuvan University in Kathmandu. A short visit of this type does not qualify a Canadian neurologist to comment on the neurological-neurosurgical problems of a country such as Nepal with any degree of expertise. Nevertheless, as a result of a number of discussions between the two authors and interviews with many other physicians, both Nepalese and Western, it was possible to form some general impressions and draw some comparisons between neurologic illnesses in Nepal and Canada.

Disorders affecting the nervous system are common in Nepal. Some of the problems which are seen regularly would be considered rare and exotic in North America. However, despite the vast differences in culture, geography, dietary habits, and socio-economic conditions, there are some surprising similarities between the patterns of neurologic illness in Nepal and Canada. Disorders such as migraine, stroke, epilepsy and meningitis are well known to Nepali physicians. To appreciate the magnitude of health care problems in Nepal and to view neurologic illness in its proper perspective, it is helpful to have a few basic facts and figures concerning the geography and population of the country.

\section{GENERAL COMMENTS}

Nepal is a small, mountainous, land-locked nation which is situated strategically between two large and powerful neighbors - India to the south and China (Tibet) to the north. By North American standards, Nepal is densely populated with approximately 15 million people occupying $143,650 \mathrm{Sq}$. Km of land, an area slightly smaller than the state of Florida. Within its borders are several of the world's highest mountains. There are 8 peaks over 8,000 meters.

Nepal, a country which was completely closed to western visitors until the 1950's, is struggling with very serious socio-economic and health problems as it attempts to develop into a modern 20th century nation. Despite substantial amounts of foreign aid, it is rated among the five poorest countries in the world. Fifty percent of children die before age 5 . The average life expectancy is still less than 50 years and only $20 \%$ of the population over age 6 can read and write.

There are only about 400 physicians in the country, the majority of whom are located in or near the capital city, Kathmandu. Few roads exist and in most parts of the country travel is entirely by foot. For many people, obtaining medical care may mean walking or being carried for several days over rough mountain trails.
With malnutrition, parasitic infestation, tuberculosis, and gastroenteritis accounting for large numbers of deaths, it is not surprising that the diagnosis and treatment of neurologic disease has not received heavy emphasis. There are no neurologists in the country and only one fully trained neurosurgeon who is based at the military hospital in Kathmandu. However, some progress is being made. As a result of Dr. Gongal's special interest in neurosurgery, a six bed neurosurgical unit has recently been established at Bir Hospital in Kathmandu. This unit deals with a variety of neurosurgical problems including 30 - 40 brain tumors a year. In addition, there is a neurology-neurosurgery outpatient clinic which provides consultation and follow-up care for approximately 100 patients a month.

\section{PROBLEMS AND LIMITATIONS}

Few figures are available concerning the incidence of various diseases, neurologic or otherwise, so it is difficult to present anything more than general impressions regarding the patterns of neurologic disorders. Furthermore, diagnostic facilities are limited and diagnoses are based largely on clinical observations. Accurate diagnosis is further restricted by the lack of pathological confirmation in the majority of cases. Surgically removed tissue is examined, but with the Hindu custom of cremation immediately following death, autopsies are seldom performed.

Cerebral angiography is carried out fairly regularly, but with rather antiquated equipment. There is no such thing as an automatic film changer, and often one must rely on a single film which may or may not have been obtained with appropriate timing to reveal the lesions. Pneumoencephalograms and ventriculograms provide an alternative means for demonstrating intracranial pathology. Myelography is also carried out but the number of procedures is limited by the prohibitively high cost of contrast material. An EEG machine and an echoencephalograph are available at Bir Hospital but neither is used with any regularity. Obviously, there is no such luxury as a CT scanner, the closest facility being at Delhi in India, approximately two hours flying time from Kathmandu. Until recently, the frequent power failures and wide voltage fluctuations in the Kathmandu electrical system would have played havoc with a CT scanner. However, a new hydro-electric project has just been completed and there is now some optimism that CT scanning may be available in the future.

\section{PATTERNS OF NEUROLOGIC AND NEUROSURGICAL ILLNESS}

Infections account for a large proportion of neurologic illness. Tuberculosis is extremely prevalent and its various neurologic manifestations are frequently encountered.

From the Department of Clinical Neurosciences, Faculty of Medicine, University of Calgary, Calgary, Alberta, Canada and the Department of Surgery, Bir Hospital, Kathmandu, Nepal.

Reprint requests to: Dr. Robert G. Lee, Department of Clinical Neurosciences, University of Calgary, Calgary, Alberta, Canada T2N 1 N4. 
Tuberculous meningitis is probably the most common form of meningitis although pyogenic and viral meningitis are also seen regularly. When a patient presents with signs of spinal cord compression, the diagnosis is Pott's disease until proven otherwise. Similarily tuberculoma must be ranked high on the list of diagnostic possibilities for a patient with signs and symptoms of a posterior fossa tumor.

Poliomyelitis is still endemic in Nepal and there is not yet any comprehensive program for immunization. At a 300 bed rehabilitation centre just outside Kathmandu, $45 \%$ of the patients are polio victims. Many of them are still children and teenagers and have obviously acquired the disease within the past few years.

Most Canadian physicians have never seen a case of human rabies. The large population of stray dogs in Kathmandu provides a reservoir for the rabies virus. At least two or three cases a year of human rabies are seen at the infectious disease hospital and these undoubtedly represent only a small fraction of the cases which occur throughout Nepal. Tetanus is a common problem. Several cases a month are seen at Kathmandu alone, many occurring as postpartum complications.

During the monsoon season from June to September, a form of encephalitis is prevalent in the Terai - the low lying subtropical region just north of the Indian border. The responsible agent has not been positively identified. Japanese B encephalitis has been suspected but this has not been confirmed. The mortality of this illness approaches $25 \%$ and a large proportion of survivors are left with permanent neurologic sequelae. Leprosy with its various neurologic manifestations affects an estimated one percent of the population. Cases of neurosyphilis are also still seen regularly.

Cretinism is endemic in the middle hills of Nepal. The incidence is estimated to be $1-2 \%$ although the figures run much higher in some villages where the incidence approaches $10 \%$. Neurologic manifestations are common and include mental retardation, deaf mutism (affecting perhaps $70 \%$ of those with untreated cretinism) and an unusual form of spastic diplegia.

An even greater concern is widespread infant malnutrition which is still common in many of the hill villages. Although the neurological effects are not so obvious and specific as those of cretinism, one cannot dismiss the fact that prolonged malnutrition occurring at a critical stage of development of the nervous system may have serious and lasting effects on higher neurologic functions.

These are some of the problems which are prevalent in an underdeveloped country, but a visiting neurologist or neurosurgeon from North America will encounter many disorders similar to those which he or she deals with at home. Stroke, while not so common as in Canada, is by no means a rare disorder. Hypertension is a common medical problem and hypertensive intracerebral hemorrhages are seen regularly. Serious head injuries are occurring with in- creasing frequency. Despite the sparsity of roads and motor vehicles, a substantial number of head injuries occur as a result of motorcycle accidents. In the rural areas, falling out of trees is a common mechanism for injuries to the brain and spinal cord. The serious shortage of fuel in Nepal requires villagers to climb to the tops of large trees in search of fire wood.

A survey of the diagnostic categories of patients seen in the neurology-neurosurgery clinic at Bir Hospital over a period of several months indicates that tension headache, migraine, and lowback pain occur at about the same rate as in a North American neurological practice. On the other hand, cervical disc problems do not seem to be particularly common despite the fact that many Nepalese spend much of their lifetime carrying heavy loads supported by tump lines on their head.

One might suspect that psychiatric problems would be rare in a country where people are not faced with the stresses and schedules of a modern industrialized nation. However, depression and psychotic disorders seem to be almost as common as in North America. Hysteria is also a surprisingly common problem, often presenting as ataxia or other disturbances of gait, aphonia, or paralysis.

Epilepsy is a well recognized disorder, and most cases at Bir Hospital are presently looked after by the psychiatric service. Parkinsonism also occurs but not commonly. Several Nepali physicians reported seeing cases which they suspected were multiple sclerosis, although the problems associated with following cases over prolonged periods makes a diagnosis of this disorder difficult.

One disease which seems to be much more prevalent than in North America is amyotrophic lateral sclerosis. Several cases were seen in the clinic during a four week period, and Nepali physicians reported that this is a common problem in practice. One wonders whether careful epidemiologic studies might reveal that Nepal is another pocket of high incidence for this particular disease.

\section{CONCLUDING REMARKS}

Disorders of the nervous system account for a substantial amount of mortality and morbidity in the people of Nepal. The provision of even rudimentary neurological and neurosurgical care to an underdeveloped nation of $15,000,000$ people with the unique geography of Nepal presents immense logistic difficulties and requires careful assessment of priorities. It is difficult to know where to begin. Certainly there is no danger that Nepal will be overpopulated by neurologists and neurosurgeons in the foreseeable future. However, the continued development of one adequately staffed and equipped neurologicalneurosurgical unit in Kathmandu would seem to be justified. Such a unit would help increase awareness of neurologic problems and would ensure that at least some of the potentially treatable conditions are recognized. 